5. Wenn er, sich nur auf das Paralysiren des Luftdruckes beschränkend, nicht geradlinig wird: bleibt die Glottis elliptisch, und es kommt kein Verschluss, sondern bloss Verengerung zu Stande. Dieser Mechanismus kann nur Falsettöne erzengen.

6. Der Unterschied im Character der Brust- and Fistelstimme, ist in der verschiedenen Gestalt der Stimmritze allein begründet.

\title{
Ueber Lecithin und Nuclein in der Hefe.
}

Von

\section{Loew.}

Wiewohl ich mich in der Kunst einer heutzutage in wissenschaftlichen Journalen wenig mehr gebräuchlichen Angriffsweise mit Hoppe-Seyler nicht messen kann, sehe ich mich doch veranlasst, noch eine kurze Mitheilung über die in Rede stehende Frage zu machen.

Hoppe-Seyler hatte bekanntlich nur auf den Phosphorsäuregehalt des ätherischen Hefeextracts hin die Anwesenbeit des Lecithin in der Hefe behauptet, was einige Bemerkungen von meiner Seite veranlasste, indem ich hervorhob, dass hier Täuschungen möglich seien; denn selbst wenn das ätherische Hefeextract nach dem Neutralisiren, Troeknen und nochmaligem Extrahiren mit absolutem Aether phosphorhaltig befunden wiirde, sollte man den Nachweis von wenigstens Cholin oder Glycerinphosphorsäure nicht versäumen, da es wohl noch andere phosphorhaltige in Aether lösliche Körper als Lecithin geben könnte. Diesen Nachweis hat Hoppe-Seyler vor einiger Zeit erbracht in einem Artikel, in dem er die heftigsten Angriffe gegen mich schleudert. Dass ich nichts verabsäumt hatte, darüber war ich mir vollständig bewusst; denn die leichte Zersetzlichkeit des Lecithins habe ich insofern beriucksichtigt, als ich stets dem etwaigen Auftreten von Cholin und Glycerinphosphorsäure während der Untersuchung meine Aufmerksamkeit schenkte. Für mich bestand nur noch die Frage: Gehört bei der mehr oder weniger sauren Reaction der Hefe ein. verhältnissmässig rasches Zerfallen des Lecithins in den Zellen zu 
den Unmöglichkeiten? - Ich verschaffte mir desshalb ganz frische, möglichst schwach sauer reagirende Presshefe und verglich dieselbe mit der gleichen Menge (1500 gr) älterer Hefe mit stärker saurer Reaction. Dabei ergab sich, dass bei erstrer in der That eine kleine Menge Lecithin durch den Cholin- and Glycerinphosphorsäure-Nachweis aufgefunden werden konnte, nachdem das ätherische Extract der lufttrocknen Hefe nach der nöthigen Reinigung mit Barytwasser gekocht wurde, - während im zweiten Falle die Reactionen mit dem ätherischen Extract so zweifelhaft ausficlen, dass man mit gutem Gewissen nicht hätte auf Lecithin schliessen dürfen. Statt des letztern mussten aber seine Zersetzungsproducte nachweisbar sein, was sich auch bestätigte. Ich hatte früher bereits diesen Zerfall in der mir zu Gebote stehenden Hefe vermuthet ${ }^{1}$ ) and desshalb dieselbe direct auf Cholin gepriff, wobei ich eine kleine Menge eines Platinsalzes erhielt, dessen Krystallhabitus allein mich zur Annahme veranlasste, es könne hier die Cholinverbindung nicht vorliegen, was ich jetzt als einen allzu hastigen Schluss erklären muss.

Es, kann bei der leichten Zersetzlichkeit durch Säuren doch gar nicht überraschen, dass selbst in ganz frischer Presshefe, wenn bei ihrer Fabrikation die Milchsäuregährung nicht sorgfältigst auf das kleinste Maass beschränkt wird, das Lecithin sich nicht in nachweisbarer Menge anbäufen kann. Eine Bierhefe ferner, die in lebhafter Gährthätigkeit begriffen ist, enthält stets saures Kaliamphosphat and auch hier kann also bei sonst sorgfältiger Arbeit ein negativer Befund leicht möglich werden.

Die leichte Zersetzlichkeit durch Säuren erklärt vielleicht ferner warum in dem von mir untersuchten Schimmelrasen Lecithin nicht nachgewiesen werden konnte; denn derselbe war auf einer Flüssigkeit gezogen worden, welche zum Zweck der Verhinderung von Spaltpilz-Entwicklung mit 1 Proc. Phosphorsäure angesäuert worden war. Wohl mag eine geringe Menge Lecithin in den rom Wasser nicht benetzbaren Sporen enthalten sein, allein diese machen häufig nur eine Minimalmenge des Totalrasens aus; in diesem Falle betrug ihre Menge kaum 1 Proe. der Mycelmasse. Bei Pilzen wie die Essigmutter, die überhaupt nur in stark sauren Flïssigkeiten leben, ist die Lecithinbildung von vornherein

1) Dies. Archiv 1879. p. 346. 
eine Unmöglichkeit. - Die Sporen von Schleimpilzen oder Mycetozoen scheinen aber geringe Mengen von Lecithin zu enthalten; denn es gelang mir, aus dem ausgezognen Fett von Aethalium septicum durch Kochen mit Barytwasser Glycerinphosphorsäure abzuscheiden. Der P-Gehalt des ausgezogenen Fettes entspräche $=0,017$ pCt. Lecithin der Sporen, voransgesetzt, dass er ganz auf letzteres bezogen werden darf.

Was nun das $\mathrm{Nuclein}$ der Hefe betrifft, so hat auch hierüber Hoppe-Seyler auf meine Bemerkungen hin nochmals Untersuchungen in seinem Laboratorium anstellen lassen. Von diesen ist der erste Theil im Juli 1879 erschienen ${ }^{1}$ ), auf die versprochene Fortsetzung aber wartete man bis jetzt vergeblich. Aus jenem ersten Theil will ich einige Sätze zur Characterisirung jenes Körpers anfuihren:

p. 288. „Es gelang trotz mehrfacher Versuche nicht, ein Präparat von demselben P-Gehalte $(6,1 \mathrm{pCt}$.) wie das analysirte wiederum herzustellen. Phosphorbestimmungen in den übrigen Präparaten ergaben 3,$28 ; 3,55 ; 3,94$ pCt. P. Diese Präparate enthielten sehr geringe Mengen von Kalk und Magnesia".

p. 289. "Kocht man Nuclein mit Wasser längere Zeit, so erhält man A) einen unlöslichen Niederschlag, B) eine wässrige Lösung, C) ein flüchtiges Product".

p. 290. „Die Zusammensetzung dieses Körpers (A) nähert sich derjenigen der Eiweisskörper".

p. 291. „Unter den löslichen Spaltungsproducten des Nucleins, deren Untersuchung noch nicht beendet ist, liess sich eine nicht unbedeutende Menge Hypoxanthin nachweisen".

Es wird also das nach der Zersetzung in Lösung Befindliche kurzweg als Spaltungsproducte des Nucleins bezeichnet, nachdem kurz vorher (p. 288) ausdrücklich gesagt wird: „Ob das so dargetsellte Nuclein ein reiner Körper ist, darüber müssen weitere Versuche entscheiden".

Nach Hoppe-Seyler zeichnet sich das Nuclein durch zwei Haupt-Eigenschaften aus:

1) Durch die Unverdaulichkeit durch Magensaft. (Dieser Beweis ist für das Hefenuclein noch nicht erbracht.)

2) Durch die Leichtzersetzlichkeit in alkalischer Lösung.

1) Zeitschft. f. phys. Chem. Bd. III, p. 284. 
Was letztere Eigenschaft betrifft, so macht sie es unumgänglich nothwendig. dass man die Stärke der zum Extrahiren der Hefe zu verwendenden Natronlösung genau angäbe. Da man im Hoppe-Seyler'schen Laboratorium einmal ein Präparat mit 6 pCt. $\mathbf{P}$ erhielt, das man nachher nicht wieder erbalten konnte, so hätte man diesem Punkte wohl etwas Aufmerksamkeit schenken können. Schon viele Autoren haben Hefe mit alkalischen Lösungen behandelt und diese dann mit Säuren gefällt, aber nie war ein Körper erhalten worden, der Phosphor in organischer Verbindung enthalten hätte. Bei zahlreichen Versuchen habe auch ich stets nur Eiweiss mit geringer Beimengung - etwa 1 pCt. - von aus Phosphaten bestehenden Aschenbestandtheilen erhalten.

Es war mir klar, dass es ganz besondere Umstände sein müssen, unter denen das "Hefenuclein" erhalten wird und ich fand in der That, dass ein Körper von den angegebenen Eigensehaften resultirt, wenn die Natronlösung nicht mehr als 2 pCt. Alkali entbält und auch in relativ nur geringer Menge - auf 1 Kilo Hefenschlamm etwa nur $1 / 2$ Liter - angewandt wird. Man rithrt den Schlamm mit der Lösung an, lässt das Filtrat direct in verdünnte Salzsäure tröpfeln und wäscht den erhaltenen Niederschlag mit sehr verdünnter Salzsäure, schliesslich mit Wasser und Alkohol. Die Ausbeute beträgt circa 7,5 pCt. der trocknen Hefe. Ich fand darin 3,70 pCt. P und nur versehwindende Spuren von Kalk und Magnesia. Mässig concentrirte Alkalilösungen zersetzen den Körper rasch, wobei Eiweiss (und Pepton?) und Phosphorsäure als Hauptspaltungsproducte auftreten.

Kocht man den frisch niedergeschlagenen Körper - nach dem Waschen mit verdünnter Salzsäure - direct mit viel Wasser, so löst sich dasselbe vollständig auf (bei wenig Wasser bleibt ein Theil des abgespaltenen Albumins ungelöst). Aus dieser Lösung fällt Kochsalz einen Eiweisskörper (in meinem Versuch $38,3 \mathrm{pCt}$ des trocknen Nucleins), während Phosphorsäure nebst einem weitern Antheil eines eiweissartigen Körpers und - wie Kossel richtig angiebt - Hypoxanthin in Lösung bleiben. Ein flüchtiges Product konnte ich entgegen den gemachten Angaben nicht wahrnehmen.

Von einigem Interesse schien es mir, die Menge des Hypoxanthins zu bestimmen; ich kochte desshalb $28 \mathrm{gr}$ des frisch gefällten gewaschenen Körpers $(-5,5$ gr Trockensubstanz) bis zur Lösung mit viel Wasser, neutralisirte mit Barytwasser, filtrirte heiss 
rom Niederschlage $a b$, behandelte das eingedampfte Filtrat mit Ammoniak und fällte das Filtrat nun mit ammoniakalischer Silberlösung. Dieser Niederschlag wurde getrocknet und gewogen und das nach der Behandlıng mit heisser Salpetersäure ungelöst bleibende, trotz Ammoniak noch beigemengte Chlorsilber subtrahirt. Das Resultat war $0,674 \mathrm{gr}$ Sarkinsilberoxyd, was für das trockue Nuclein einen Gehalt von 5,6 pCt. Sarkin giebt. Das aus der heissen Salpetersäure auskrystallisirende Sarkinsilbernitrat diente $\mathrm{zu}$ einer Silberbestimmung; diese lieferte 31,5 p Ct. Ag, statt 29,8, was auf noch beigemengte Unreinigkeiten schliessen lässt.

Ist nun der aus Hefe gewonnene Körper ein Individuum oder ein Gemenge? - Dass im erstern Falle ein interessanter Körper vorliegen mîisste, eine - vielleicht der Distearylglycerinphosphorsäure ähnlich constituirte - Sarkinalbuminphosphorsäure ${ }^{1}$ ), wird sich Hoppe-Seyler wohl selbst gesagt haben. Da Sarkin in verdünnten Säuren nicht unlöslich ist, so liesse sich dieses als Beweis für die Behauptung, dass es dem Niederschlag nicht bloss beigemengt sei, vielleicht anführen. Unter diesen Umständen bliebe die Vernachlässigung des Studiums einer so interessanten Verbindung um so auffälliger, wenn damit nicht zugleich das Geständniss verbunden wäre, dass hier ein Körper vorläge, der von allen bisher untersuchten "Nucleinen" abwiche. In einem anderen Falle - dem Nuclein der Milch - ergaben sich zwar auch Phosphorsäure und ein Eiweisskörper als Spaltungsproducte, jedoch das Sarkin ist noch nicht als Spaltungsproduct aus Nucleinen anderer Quelle als Hefe erhalten worden ${ }^{2}$;.

Lubavin ${ }^{3}$ ) versuchte die Synthese jenes Milchnucleins ans Casein und phosphorsaurem Natrium, was voraussichtlicherweise nicht zum gewünschten Ziele führen konnte. Uebrigens ist jener Körper, wie Lubavin selbst zugiebt, ein Gemenge.

Da die Eiweisskörper sowohl Amido- als Hydroxyverbindungen sind, so können sie sich in zweierlei Weise mit Phosphorsäure verbinden, in einer lockern, nach Art der Amidosäuren ${ }^{4}$ )

1) Wie Schade, dass eine solche mehr Sarkin erfordern würde!

2) Dass Mieschers Lachssperma-Präparate Sarkin und Guanin als Verunreinigung enthalten, "hat Picard (Jahresb. 1874) gezeigt. Meiner Meinung nach ist auch beim Hefenuclein das Sarkin nur beigemengt.

3) Ber. D. Chem. Ges. 10. 2237.

4) Ein solches phosphorsaures Albumin hat Johnson dargestellt. Chem. soc. journ. [2]. 12. 
und in einer festern, nämlich unter Wasseraustritt, wie der Fall bei Glycerinphosphorsäure. Auf die im letztern Falle entstehende Albaminphosphorsäure scheint das Milch- und Hefenuclein zurüekzaführen za sein.

Da wie ich frïher gezeigt habe '), aus Albumin und concentrirter Schwefelsäure sich eine Albuminschwefelsäure herstellen lässt, so lag es nahe, die Darstellung einer Albuminphosphorsäure in analoger Weise zu versuchen. Bei gewöhnlicher Temperatur wirkt nun selbst nach längerer Zeit syrupöse Phosphorsäure kaum auf Albumin ein. Erwärmt man aber feingeriebenes Albumin mit dem etwa 10fachen Gewicht der syrupösen Säure ganz kurze Zeit auf $70-80^{\circ}$, mischt mit Wasser, und lässt das Ungelöste nach dem Waschen mit Wasser mit sehr verdünnter Natronlösung stehen bis es gelöst ist und fällt das Filtrat mit Salzsäure, so hat man einen Eiweisskörper, der Phosphorsäure in festerer Verbindung enthält und das Verhalten mancher Nucleine zeigt. Die Ausbeute ist wegen gleichzeitiger weiterer Einwirkung bei der Darstellung gering. Ich hoffe jedoch, die Darstellungsmethode zu verbessern und daruiber dann weitere Mittheilungen zu machen.

Schliesslich seien mir noch einige andere Bemerkungen gestattet. Hoppe-Seyler wirft mir vor, ich hätte „die Entdeckung der Bayrischen Academie der Wissenschaften mitgetheilt, dass Nuclein gar nicht existirte ${ }^{2}$ )!" Hier liegt wohl ein nicht ganz unabsichtliches Missverständniss zu Grunde. In meiner Mittheilung ${ }^{3}$ ) heisst es wörtlich: „Nach einer Angabe Hoppe-Seyler's kommt in der Hefe trotz des Mangels eines Zellkernes doch dieselbe Substanz vor, aus welcher die Kerne der Blut- und Eiterkörperchen bestehen und welche man Nuclein nannte. Trotzdem schon von mehreren Sciten die Individualität des Nucleins in Frage gestellt wurde, versuchte ich die von Hoppe Seyler gemachten Angaben zu prïfen." Kann man das wohl als Mittheilung der "Entdeckung" ansehen, dass Nuclein gar nicht existirte? Nur über das der Hefe hatte ich mich direct dahin ausgesprochen, dass hier im Wesentlichen ein unreiner Eiweisskörper vorliege, während über das des Eiters und Spermas ich selbst kein Urtheil gefällt

1) Journ. f. pr. Chem. [2]. 3.

2) Ztschr. f. physiolog. Chem. III. p. 376.

3) Sitzungsber. d. Bayr. Acad. d. Wiss. Mai 1878. 
habe, sondern nur die von Andern bereits geäusserte Ansicht, dass hier Gemenge vorlägen, erwähnte.

Und keine Zweifel an der Individualität eines Körpers äussern dürfen, dessen P-gehalt von 2-9 pCt. wechselnd befunden wurde, der bald Eiweissstoffe bei seiner Spaltung liefert, bald gar nichts mit diesen zu thun hat (wie das Nuclein des Lachsspermas) heisst denn doch allzustarke Zumuthungen an den Autoritätsglauben gemacht. Freilich sah sich Hoppe-Seyler später selbst zur Annahme gedrängt, es gäbe mehrere $\mathrm{Nucleine,} \mathrm{allein} \mathrm{das} \mathrm{hindert}$ ihn nicht, dass er in seinem Laboratorium noch immer bei Nucleinberechnungen aus einer gefundenen Phosphorsäuremenge die Formel des Lachssperma-Nucleins zu Grunde legen lässt. So wurde das Nuclein im Menschenhirn auf folgende Weise bestimmt ${ }^{1}$ ): Das Hirn wurde erst mit Aether und Alkohol, dann mit Wasser extrahirt und hierauf mit Zusatz von Baryumcarbonat eingeäschert, dann zur Asche Schwefelsäure behufs Entfernung des Baryts gesetzt, das Filtrat mit Ammoniak neutralisirt und nun in der von den Phosphaten abfiltrirten Fliissigkeit die noch vorhandene Phosphorsäure bestimmt und aus dieser nach der Formel des Nuclein des Lachs, sperma der Nucleingehalt des Menschenhirns berechnet, ohne den leisesten Beweis für eine solche Identität.

1) Zeitschrift f. physiolog. Chem. I. 334. 